Revista Calidad en la Educación Superior

Programa de Autoevaluación Académica

Universidad Estatal a Distancia

ISSN 1659-4703

Costa Rica

revistacalidad@uned.ac.cr

\title{
PROPUESTA DE CREACION DE UN CENTRO DE ATENCION INTEGRAL AL ESTUDIANTE DE LA UNED (CEATIE) \\ PROPOSAL TO CREATE AN INTEGRAL ATTENTION CENTER FOR THE STUDENT OF THE UNED (CEATIE)
}

\author{
Ariana Acón Matamoros ${ }^{1}$, aaconm@uned.ac.cr \\ Javier Cox Alvarado ${ }^{2}$, jcox@uned.ac.cr \\ Universidad Estatal a Distancia de Costa Rica
}

\author{
Volumen 5, Número 1 \\ Mayo 2014 \\ pp. $205-222$
}

Recibido: noviembre, 2013

Aprobado: abril, 2014

\footnotetext{
${ }^{1}$ Máster en Gerencia de Negociaciones Internacionales, Licenciada y Bachiller en Informática. Labora actualmente en el Programa de Autoevaluación Académica de la Universidad Estatal a Distancia de Costa Rica como asesorainvestigadora.

${ }^{2}$ Doctor en Educación. Director de la Revista Electrónica Calidad en la Educación Superior (CAES). Actualmente labora como coordinador del Programa de Autoevaluación de la Universidad Estatal a Distancia de Costa Rica.
} 


\section{Resumen}

Se detalla una propuesta para la creación de un centro de atención integral al estudiante de la Universidad Estatal a Distancia (UNED), que reúna los componentes administrativos y tecnológicos, enfocados en atención al cliente, para brindar una respuesta de calidad a los estudiantes.

La importancia de la creación del centro de atención integral al estudiante se describe en el desarrollo de esta investigación, y los beneficios inmediatos para los estudiantes y la universidad.

A su vez una mejora en el servicio que se presta al estudiantado, representa el mejoramiento continuo, repercute en la calidad de la educación superior que brinda la universidad a sus estudiantes, apoyado por los sistemas de información con los que se cuenta y los procesos de autoevaluación y acreditación de la UNED.

Palabras clave: atención integral, servicio al estudiante, calidad en los servicios, calidad en la educación superior.

\section{Abstract}

A proposal for the creation of the student integral attention for the Universidad Estatal a Distancia (UNED), satisfying the administrative and technological components, focused on customer service to provide quality feedback to students is detailed.

The importance of the creation of the student integral attention is described on this research and the immediate benefits for students and the university.

At the same time an improvement in the service provided to students, represents the continuous improvement and makes an impact in the quality of higher education that the university provides to its students, supported by the information systems that already exists and the self-assessment and accreditation process of the UNED

Keywords: integral attention, student services, services quality, higher education quality. 


\section{INTRODUCCION}

La Universidad Estatal a Distancia (UNED, 2014) fue creada con la misión de llevar la educación superior a personas con dificultad para cursar una carrera en los sistemas tradicionales, ya sea por motivos de distancia geográfica 0 personales (p.4). Una de sus características era que la población estudiantil normalmente eran adultos que trabajan quienes no habían tenido la oportunidad de asistir a una universidad tradicional.

Sin embargo, con el pasar del tiempo y en la actualidad, según la experiencia vivida en el cuido de exámenes de la universidad y de visitas a centros universitarios, es notoria la tendencia cada vez más marcada a que estudiantes más jóvenes sean quienes están matriculando en la actualidad. La UNED (2012) es la segunda universidad en cuanto al número de población en Costa Rica por lo que debido a ese crecimiento, también crece el número de consultas que se realizan a la institución. (p.3).

Con base en las estadísticas institucionales, proporcionadas por el Centro de Investigación y Estadística Institucional, se pude comprobar que el grueso de la población estudiantil se encuentra en un rango de edad no superior a los 30 años, tal y como se puede observar en el siguiente cuadro:

Tabla 1. Estudiantes matriculados en grado, por sexo según grupo de edad I Cuatrimestre 2014

\begin{tabular}{|l|c|c|c|}
\hline \multicolumn{1}{|c|}{ GRUPO DE EDAD } & HOMBRES & MUJERES & TOTAL \\
\hline Menos de & 675 & 1190 & 1865 \\
\hline De 20 a menos de 25 & 2085 & 4193 & $\mathbf{6 2 7 8}$ \\
\hline De 25 a menos de 30 & 1864 & 3741 & $\mathbf{5 6 0 5}$ \\
\hline De 30 a menos de 35 & 1352 & 2289 & 3641 \\
\hline De 35 a menos de 40 & 806 & 1359 & 2165 \\
\hline
\end{tabular}




\begin{tabular}{|l|c|c|c|}
\hline De 40 a menos de 45 & 421 & 658 & 1079 \\
\hline De 45 a menos de 50 & 264 & 403 & 667 \\
\hline De 50 a más años & 261 & 352 & 613 \\
\hline Sin ubicar & 3 & 8 & 11 \\
\hline TOTAL & $\mathbf{7 7 3 1}$ & $\mathbf{1 4 1 9 3}$ & $\mathbf{2 1 9 2 4}$ \\
\hline
\end{tabular}

Fuente: Centro de Investigación y Evaluación Institucional, con datos obtenidos del Sistema de Administración de Estudiantes (SAE) el 24/03/2014

Con base en lo anterior, es notorio que la población estudiantil ha sufrido un cambio en relación con sus características, especialmente etarias. Asimismo por ser la UNED la universidad pública cuyos aranceles en relación al costo de matrícula y de asignaturas sea uno de los más bajos del país, es natural que crezca en cuanto a población.

Debido a ese crecimiento, es normal que aumente la demanda de servicios que ofrece la universidad a sus estudiantes. Según Rojas y Acón (2010) uno de los criterios de la gestión académica para la calidad es el conocimiento en cuanto a los aspectos del quehacer académico del estudiante, es decir la información. La propuesta de creación de un centro integral al estudiante, es precisamente, ofrecer esa información de manera inmediata para mejorar el servicio que se brinda al estudiantado.

\section{Propuesta de creación de un centro de llamadas}

El Programa de Autoevaluación Académica, implementó un plan piloto para desarrollar la evaluación sistemática virtual de las asignaturas en el 2010 con miras a una implementación permanente (Rojas y Acón) por medio de encuestas que se realizan una vez al cuatrimestre mediante una plataforma en línea que envía invitaciones por correo electrónico a los estudiantes, por tal motivo solicitó a la DTIC un correo en donde se pudiera recibir notificaciones de los estudiantes. El correo es evaluación@uned.ac.cr, como en evaluación de cursos. (p.72) 
Sin embargo, son muchos los estudiantes que confunden el correo y envían sus consultas al mismo, como las que se observan a continuación en el cuadro 2 :

\section{Cuadro 2. Consultas de estudiantes al correo evaluación de la UNED ${ }^{3}$}

\section{De: Estudiante 1}

Enviado el: martes, 01 de abril de 2014 10:02 a.m.

Para: evaluacion@uned.ac.cr

Asunto: Evaluación de administración 2

Hola, mi nombre es XXXXXXX, pude ver que la materia de administración me sale en el perfil que la perdí pero eso no es cierto yo hice la prueba en Ciudad Quesada y gane el examen ruego que me revisen esa nota.

Muchas gracias.

\section{De: Estudiante 2}

Enviado el: viernes, 21 de marzo de 2014 02:10 p.m.

Para: evaluación

Asunto: RE: Recordatorio para participar en una encuesta, UNED, Acreditación y Autoevaluación de carreras, Evaluación de Cursos Escuela de Administración.

Buenas tardes!

Deseo hacer una observación.

Los Encargados de Programa y Cátedra a mí criterio deben revisar los libros que los estudiantes debemos estudiar, ya que por ejemplo en la materia Sistemas de Información Gerencial para el primer examen se debe estudiar 8 capítulos de los cuales se puede suprimir algunos temas que son obsoletos. Comprendo que el Programa de Administración en RRHH está en periodo de transición.

Gracias

\footnotetext{
${ }^{3}$ Se omite el nombre, correo de los estudiantes y se corrigen algunos errores ortográficos, por motivo de presentación en este artículo.
} 


\section{De: Estudiante 3 \\ Enviado el: miércoles, 05 de marzo de 2014 09:06 p.m. \\ Para: evaluacion@uned.ac.cr \\ Asunto: No acordes}

Creo que el tipo de exámenes no son apropiados, para nosotros los estudiantes a distancia. Son muy detallistas con las preguntas. No es posible que un examen tenga 14 páginas. Creo que con esto; pareciera, que la UNED lo que busca es que haya bastante matrícula, cada semestre y no que el estudiante aprenda y apruebe los cursos.

\section{De: Estudiante 4}

Enviado el: jueves, 16 de agosto de 2012 17:38

Para: evaluacion@uned.ac.cr

Asunto: consulta

Quisiera dirigirme a los encargados de valuación de la materia Psicología de la motivación, código 989. Mi nombre es XXXXXXX. Soy estudiante de la carrera educación preescolar en la UNED de Turrialba. Quisiera comunicarles que durante el I cuatrimestre del presente año curse esta materia la cual reprobé y tuve que realizar examen de reposición, en el cual me fue muy bien por lo que la materia la aprobaba, pero en el historial académico se registra como perdida, envié la apelación correspondiente y fue aprobada, pero al día de hoy la materia aún sigue registrada como perdida.

Les pido de favor que me resuelvan el asunto del promedio final, ya que esta vez es la segunda ocasión que llevo la materia y por un error de estos no me gustaría tener que llevarla otra vez.

Muchas Gracias!!

Fuente: elaboración propia, con datos del correo evaluación@uned.ac.cr, 2014

Los cuatro correos que se toman como ejemplo reflejan las diferentes inquietudes de los estudiantes como son:

1. Error en la calificación

2. La actualización del texto de los libros,

3. La materia a cubrir para un examen (8 capítulos),

4. Lo extenso de los exámenes (14 páginas) en donde opina la estudiante que la UNED busca bastante matrícula pero que no aprueben los estudiantes, y

5. Errores en el historial académico.

Correos como esos se reciben todos los días, y lo hacen por medio de este correo evaluación@uned.ac.cr que debiera ser sólo para consultas relacionadas a la 
evaluación de cursos de carreras acreditas o en proceso de autoevaluación para acreditación, por cuatrimestre; lo que a su vez indica que los estudiantes desconocen como canalizar sus consultas en forma correcta. Es por lo que a su vez sugiere la necesidad de innovar con el apoyo de la tecnología al crear un centro de atención integral al estudiante de la UNED.

Cárdenas (2012), en relación con el servicio al cliente en instituciones educativas señala lo siguiente:

Por esta razón no es raro que las instituciones educativas hayan desarrollado cierto desdén hacia las motivaciones y deseos de sus clientes. De manera vertical, en un escenario que lo permitía, el cliente académico directo (entiéndanse estudiantes y padres de familia) debían aceptar sin mayor prejuicio las posturas de la institución. Era eso o perder el cupo sin ningún miramiento.

Desde esos anales se malversó el tema de la calidad académica con la prepotencia y la antipatía propia de las instituciones educativas prestigiosas de la época.

Los anteriores ejemplos, con base en los correos recibidos, dejan clara que la aseveración antes expuesta se encuentra vigente en nuestra institución y permite reflexionar sobre las necesidades que tienen los estudiantes de contar con un servicio al cliente que acorde a los criterios de calidad en las organizaciones, satisfaga sus necesidades.

\section{Planteamiento del problema.}

Con base en lo anterior, se plantea el siguiente problema:

¿Cómo se puede realizar una propuesta para la creación de un centro de atención integral al estudiante de la UNED (CEATIE) con el fin de centralizar las consultas telefónicas y por medio de correo electrónico, para dar una respuesta rápida, eficiente y real, utilizando los recursos con los que ya cuenta la universidad? 


\section{Objetivo General}

1. Realizar una propuesta para la creación de un centro de atención integral al estudiante de la UNED (CEATIE) con el fin de centralizar las consultas telefónicas, por medio de correo electrónico y otros, para dar una respuesta rápida, eficiente y real, utilizando los recursos con los que ya cuenta la universidad.

\section{Objetivos específicos}

1. Detallar los recursos necesarios para la creación del CEATIE, con el fin del establecimiento de los que existen y lo que se necesitan para su funcionamiento óptimo.

2. Establecer una propuesta de diseño para el CEATIE que contemple los recursos establecidos, el personal necesario y la infraestructura requerida entre otros.

3. Determinar los costos financieros necesarios para el funcionamiento del CEATIE, con el fin de demostrar su factibilidad.

4. Proponer un plan de acción con el fin del establecimiento del CEATIE a corto plazo.

5. Determinar los beneficios para los estudiantes y la universidad derivados de la creación del CEATIE.

\section{Marco conceptual}

\section{Acuerdo de Mejoramiento Institucional}

En el sitio Web (UNED, 2014) se establece la suscripción del Acuerdo de Mejoramiento Institucional (AMI) para la ejecución del Plan de Mejoramiento Institucional de la universidad.

El mismo contempla el Plan de Mejoramiento Institucional (PMI), en donde se establece que:

Las estrategias de inversión, tendrán un impacto directo en la creación de una red de Centros Universitarios para la innovación, el desarrollo local y 
nacional, en el desarrollo de recursos didácticos digitales y el aprovechamiento de las tecnologías de la información y comunicación, así como en la diversificación de la oferta académica y la formación y la capacitación en el modelo de educación a distancia. (UNED, 2014)

Se observa, que la innovación y el aprovechamiento de las tecnologías de la información y comunicación, es parte importante del PMI, por lo que la propuesta para el Centro de Atención de Llamadas refuerza esa estrategia.

\section{Plan de Mejoramiento Institucional de la UNED}

El Plan de Mejoramiento Institucional se encuentra disponible en el sitio Web de la UNED, y es parte del AMI y se aprecia como eje estratégico:

...ejes estratégicos del Plan de Desarrollo de la UNED (2012), se apuesta a una estrategia de fortalecimiento inteligente y sostenido de las TIC en su modelo educativo, que permita modernizar tecnológicamente los procesos administrativos $y$ académicos $y$ responder a las necesidades $y$ requerimientos de la comunidad universitaria y del país, de manera oportuna y pertinente, de acuerdo a los sistemas más modernos de enlace y acceso en redes. (p.8).

EI CEATIE apoya la modernización tecnológica de los procesos administrativos y académicos, y también es una estrategia de servicio al cliente, usuario que en este caso es el estudiante, la razón de ser de la universidad.

El servicio ágil, oportuno y eficiente al estudiante no se contempla en el AMI, ni en el PMI, pero es una pieza fundamental para los años venideros.

\section{Calidad en la Educación Superior}

Según Cox (2011), citando a Lemaitre (2008) “...el aseguramiento de la calidad se debe trabajar con las instituciones y con los responsables del desarrollo de programas..." 
De tal manera:

que la educación superior cumpla con la función que le asigna la sociedad: garantizar que una proporción cada vez mayor de la población adquiera las capacidades fundamentales para su desarrollo personal y para el desarrollo nacional, lo que va mucho más allá de lo que solía ser la responsabilidad de la educación superior. (p.214)

Aunado lo anterior a la misión de la UNED de ofrecer educación superior a todos los sectores de la población (UNED 2014) y para ello hace uso de los medios tecnológicos que permiten la interactividad, e aprendizaje independiente. Además se compromete con la excelencia académica entre otros, para la construcción de una sociedad justa y una cultura de paz.

Con lo expuesto hasta ahora, se justifica la creación del CEATIE como una innovación que va de la mano con las tecnologías de información, que buscan apoyar la excelencia académica y por ende la calidad en la educación superior.

\section{Recursos disponibles}

Los recursos disponibles con que cuenta la universidad son los sistemas de información transaccionales que ya existen y que registran la información de los estudiantes o con relación a ellos, como ejemplo se pueden citar:

1. SAE: Sistema de Administración de Estudiantes

2. Notas Parciales

3. Cargas Académicas

4. Apelaciones

5. graduaciones

6. Becas

7. Consultas estudiantes vía Web

8. Matrícula Web (Matriweb)

9. Matrícula presencial 
También la universidad cuenta con espacio físico, equipo de cómputo y personal que se puede asignar al CEATIE.

\section{Centralización de consultas telefónicas y por correo}

En la universidad funciona el Programa de Atención Didáctico a Distancia (PADD), que atiende consultas específicas de las asignaturas, de la población estudiantil, y brinda respuesta en un periodo establecido por la administración. Los horarios varían de acuerdo a la semana, y se enmarcan de lunes a viernes de 8:00 a.m. a 4:30 p.m. Además cuenta con un buzón telefónico del PADD, en el cual se deja la consulta grabada y por ese mismo medio se obtienen la respuesta, en un plazo máximo de 24 horas.

En el entorno estudiante de la página web en la dirección http://intranet.uned.ac.cr/entornoestudianti//login.aspx, se puede realizar diferentes consultas como son historial académico, historial de becas, condicionamiento, morosidad, historial de solicitudes, graduación, préstamos biblioteca, historial de préstamos biblioteca, Centros Universitarios, notas parciales, horarios de tutorías. Esta facilidad representa una ayuda para los estudiantes, siempre y cuando cuenten con acceso a internet y a una computadora.

En el entorno estudiantes, está a disposición los siguientes formularios:

Encuesta de graduados, solicitud de certificación, solicitud de estudio y graduación, defensoría de los estudiantes (electrónico), respuesta de tareas, solicitud de apelación, solicitud de examen calificado y solicitud de traslados. 


\section{Figura 1. Entorno Estudiantil}

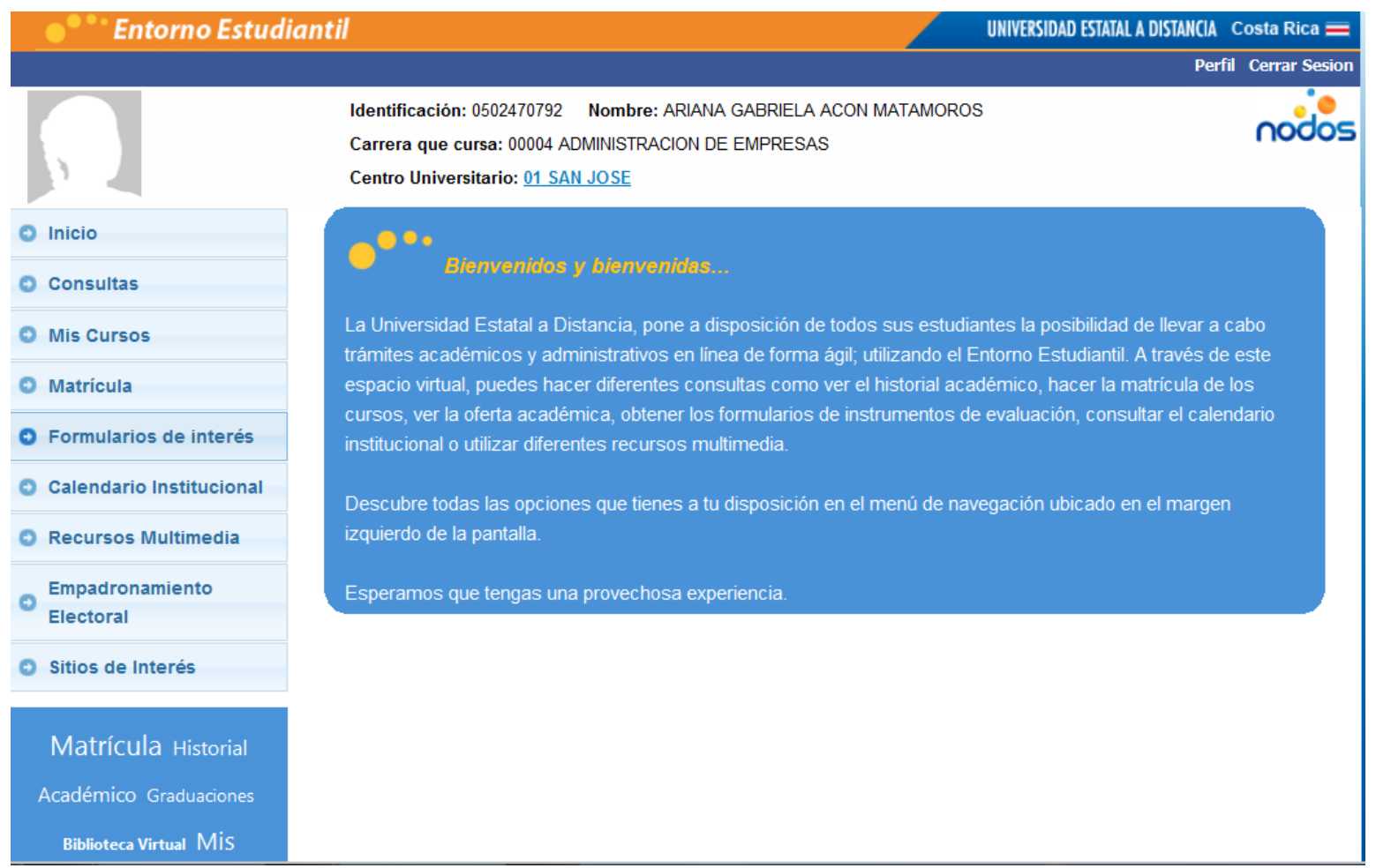

Fuente: página Web de la UNED, 2014

\section{Descripción de los recursos necesarios para la creación del CEATIE}

Los recursos necesarios existentes se describen en el apartado Recursos Disponibles y Entorno Estudiantil. Además entre los necesarios está un Sistema de Información que integre las consultas de todos esos otros sistemas de información, centralizados en una sola aplicación informática para que el operador de un Centro de Llamadas (mayormente conocido con su nombre en inglés: Call Center) pueda responder de inmediato y de forma ágil las consultas que se realicen, ya sea por teléfono o por correo electrónico. 


\section{Propuesta de diseño para el CEATIE}

\section{Como se puede utilizar esos recursos para ponerlo en funcionamiento en el CEATIE?}

Para la realización de la propuesta de diseño e implementación del CEATIE es necesario la colaboración de la Dirección de Tecnologías de Información y Comunicación (DTIC), con la asignación de un líder de proyectos y dos analistas de sistemas que puedan desarrollar una aplicación para Web, con interfaz amigable para la realización de las consultas. Se sugiere la utilización de Punto Net como herramienta de desarrollo, pues la universidad ya cuenta con las licencias para su uso además de ser una herramienta muy utilizada para los desarrollos Web.

Sería conveniente contar con una base de datos con las preguntas frecuentes de tal manera que al digitar la pregunta del estudiante, ya existan algunos ejemplos dados de cómo resolver esas dudas.

\section{Recursos necesarios}

\section{Personal}

Para la implementación del CEATIE se necesita como mínimo a tres personas a tiempo completo que tengan el siguiente perfil:

- Bachiller en Educación Media

- Dos años de estudios en una carrera de Informática, Administración de Empresas, o similar

- Manejo de Ofimática

- Experiencia en el manejo de conflictos

- Preferiblemente con manejo del idioma inglés u otro 


\section{Infraestructura requerida}

\section{Organizacional}

Para determinar su lugar en el organigrama institucional, es necesario realizar un estudio aparte, que determine cuál sería la mejor oficina, o dependencia que pueda supervisar las labores del CEATIE.

\section{Física}

Oficina para tres personas como mínimo equipada con mobiliario, computadoras modernas, central telefónica, la cual puede funcionar por medio de Internet (IP), para no saturar la central de la UNED.

\section{Hardware}

El hardware necesario en esta etapa son las computadoras equipadas con Windows, Office y el sistema de información de respuestas a los estudiantes por desarrollar.

\section{Software}

El software que se requiere es el que debe desarrollar la DTIC, específico para la solución inmediata a las consultas de los estudiantes.

\section{Proyección de los costos financieros necesarios para el funcionamiento del CEATIE}

Para la implementación del CEATIE después de la primera etapa de desarrollo del sistema de información por el personal de la DTIC para la respuesta inmediata a los estudiantes, se requiere de tres personas que darían el soporte al usuario final (estudiante) por medio del Centro de Llamadas, a las cuales habría que cubrir su salario y cargas sociales que esto implica. 


\section{Propuesta de un plan de acción con el fin del establecimiento del CEATIE a corto plazo}

Para poder establecer el funcionamiento del CEATIE, es necesario elaborar la propuesta y elevarla para su aprobación al Consejo Universitario, tomando en consideración el análisis financiero y la infraestructura requerida.

Para ese fin según lo visto en el apartado de la propuesta, es recomendable utilizar personal disponible de la universidad que ya cuenta con conocimiento necesario para empezar.

\section{Determinar los beneficios}

\section{Para los estudiantes}

Una visión del beneficio que representaría para el estudiante la creación del CEATIE, es un solo número telefónico gratuito 800 o 900 con una central aparte, que tenga capacidad para atender 20 llamadas por minuto, en donde se responda de manera inmediata al estudiante su inquietud. En caso de no poder dar respuesta inmediata, el funcionario del CEATIE debe canalizar la misma con el canal oficial requerido y darle seguimiento, para ofrecer la respuesta al estudiante en el menor tiempo posible.

A continuación se listan otros beneficios para los estudiantes:

- Un sistema de información que resuelve de manera inmediata las consultas del estudiante como notas, apelaciones, históricos, entre otros.

- Solicitar servicios como constancias que emite la Oficina de Registro.

- Canalizar las consultas que no se pueden resolver y darle seguimiento para la repuesta.

- Exponer sus quejas o disconformidad con el fin de darle seguimiento y enviarle notificación a la Defensoría de los Estudiantes.

- Escuchar, registrar y dar trámite a las sugerencias con el fin del mejoramiento continuo. 


\section{Para la universidad}

Esta propuesta pretende reutilizar los recursos con los que cuente la UNED en cuanto a personal, infraestructura, equipo de cómputo, sistemas de información.

Es importante visualizar los beneficios que su puesta en práctica implicaría:

- Brindar un servicio al estudiante de acuerdo con la excelencia académica de la universidad.

- Resolver las consultas de los estudiantes de manera sincrónica y asincrónica.

- Podría resultar en asesoría para acortar el tiempo de permanencia del estudiante en la universidad.

- Podría ayudar a disminuir el porcentaje de deserción estudiantil.

- Mejora del servicio que se brinda al estudiante.

- Incremento del grado de satisfacción del estudiante con relación a los servicios que brinda la universidad.

- Resolución de conflictos de forma amigable y en un periodo corto.

\section{CONCLUSIONES}

- El presente artículo ha visualizado los múltiples beneficios que conlleva la puesta en práctica del CEATIE tanto para la universidad como para los estudiantes.

- Como se ha podido observar, es una la propuesta realista, la cual no genera grandes inversiones y sobre todo necesaria para una gestión de calidad a nivel institucional.

- La existencia de un Centro de Llamadas cuyo enfoque sea el servicio al cliente, que en el caso de esta institución son los estudiantes, podría mejorar en general la imagen de la UNED, que conlleva una satisfacción en el estudiantado y contribuye al mejoramiento continuo de la institución. 
- La propuesta de la creación de un Centro de Llamadas, es una iniciativa que promueve el trabajo entre dependencias, lo cual podría desterrar ciertas prácticas como el trabajo aislado y la duplicidad de funciones.

- Es importante socializar esta propuesta con la finalidad de que sea estudiada por las autoridades y pueda tornarse en una realidad.

\section{REFERENCIAS BIBLIOGRAFICAS}

Cárdenas, L. (s.f.). Acerca del servicio al cliente y "otros demonios" de las Instituciones Educativas. Recuperado de http://mercadeoeducativocolombia.blogspot.com/2012/01/acerca-delservicio-al-cliente-y-otros.html

Cox, J. (2011). Opinión de empleadores sobre la formación de los graduados de la carrera de Administración de Empresas con énfasis en Contaduría de la Universidad Estatal a Distancia (UNED) de Costa Rica: un insumo fundamental para el aseguramiento de la calidad de la Carrera. Revista Electrónica Calidad en la Educación Superior. 2(2). Recuperado de http://web.uned.ac.cr/revistas/index.php/caes/article/view/73/124

Rojas, M. y Acón, A. (2010). Evaluación de cursos por parte de los estudiantes: un insumo indispensable para una gestión académica de calidad. Revista Electrónica Calidad en la Educación Superior 1(2).Recuperado de http://web.uned.ac.cr/revistas/index.php/caes/article/view/73/124

Universidad Estatal a Distancia UNED. (2012). Plan de Mejoramiento Institucional. Recuperado de http://www.uned.ac.cr/index.php/ami/documentos/documentos-ami

Universidad Estatal a Distancia UNED. (2014). Misión y Visión. Recuperado de http://www.uned.ac.cr/index.php/rectoria/myv/19-rectoria/118-mision-de-launed

Universidad Estatal a Distancia UNED. (2014). Acuerdo de Mejoramiento Institucional. Recuperado de http://www.uned.ac.cr/index.php/ami/inicio

Universidad Estatal a Distancia UNED. (2014). Plan de Mejoramiento Institucional. Recuperado de http://www.uned.ac.cr/index.php/ami/inicio/128-ami/607-ami 
Vicerrectoría de Planificación. Centro de Investigación y Evaluación Institucional de la UNED. (2014). Estadísticas de Matrícula I Cuatrimestre 2014. Recuperado de http://www.uned.ac.cr/viplan/images/ciei/estadisticas/ESTADISTICAS_DE_ MATRICULA_I_Cuatrimestre_2014.pdf 